\title{
INFLUENCE OF FRINGING ON SERVOVALVE TORQUE-MOTOR CHARACTERISTIC
}

\author{
Eizo URATA \\ Department of Mechanical Engineering, Faculty of Engineering \\ Kanagawa University \\ Rokkakubashi 3-27-1, Kanagawa-ku, Yokohama, 221-8686 Japan \\ (E-mail: uratae01@kanagawa-u.ac.jp)
}

\begin{abstract}
Servovalve torque-motors are controlled changing magnetic flux through air gaps at the armature ends. The traditional theory gives the torque on the armature assuming uniform distribution of the flux over the air gaps. However, experimental facts contradict with the assumption because the flux density becomes uneven by the fringing at the gaps. To express the torque with acceptable accuracy, two coefficients are necessary. The first is the ratio of the mean flux density and the flux density at the center of the gap. The second is the ratio of mean of squared flux density and the square of the flux density at the center of the gap. This paper explains the necessity of these coefficients and presents the procedure to find them. The obtained result forms a part of design criteria for servovalve torque-motors.
\end{abstract}

\section{KEY WORDS}

Servovalve, Torque-motor, Fringing, Flux density distribution

\section{NOMENCLATURE}

$A_{i}(i=1,2,3,4)$ : Sectional areas of poles.

$A_{g}$ : Cross section of air gap.

$B_{C i}(i=1,2,3,4)$ : Flux density at the centers of gaps.

$B_{m i}(i=1,2,3,4)$ : Mean value of flux density on $S_{i}$.

$B_{T_{i}}(i=1,2,3,4)$ : Root mean square of flux density on $S_{i}$.

$B(x, y)$ : Flux density at point $(x, y)$

$F_{1}$ : Attractive force between pole-1 and armature

$I_{B}(y)$ : Flux density factor defined by (12).

$I_{T}(y)$ : Square of flux density factor defined by (12).

$J_{B}(x)$ : Flux density factor defined by (13).

$J_{T}(x)$ : Square of flux density factor defined by (13).

$k_{m}$ : Coefficient related to flux defined by (8).

$k_{T}$ : Coefficient related to torque defined by (8).

$L_{0}$ : Distance between flexure tube center and pole center
$M_{1}$ : Moment due to magnetic force $F_{1}$

$T_{A}$ : Torque generated by magnetic attractive forces

$\mu_{a}$ : Permeability of air

$\xi$ : Distance from flexure tube center

$\Phi_{\imath} \quad(i=1,2,3,4):$ Flux through gap $i$.

\section{INTRODUCTION}

The servovalve torque-motor considered in this paper is used for the first stage amplifier of two-stage servovalves. Figure 1 illustrates the structure and the components of the torque-motor[1]. In Fig.1, a flapper stands at the center of the torque-motor. At the upper end of the flapper a prismatic bar, called armature, made of soft magnetic iron is fixed. The flapper threads through a flexure tube. The upper end of the flexure tube is welded 
to the armature. The lower end of the flexure tube is built into the valve body that forms the base of the torquemotor construction. At the lower end of the flapper, a pair of nozzles is located.

When the armature deflects, the lower end of the flapper is displaced and the gaps between the nozzles are changed. The change of the nozzle gaps induces the change of the fluid pressures in the nozzles. In this way, the armature deflection controls the nozzle pressures that drive the main spool of the servovalve.

To deflect the armature, magnetic components are used. As shown in Fig.1, a pair of yokes, upper and bottom, are connected by two parallel oriented identical permanent magnets (only one magnet is shown). Thus, the ends of the yokes become magnetic poles. The poles at the ends of the upper yokes are N-poles; the poles of the lower yokes are S-poles. The geometric dimensions of the yokes and the armature are determined to form small gaps between the poles and the armature surface. Two coils are put on the armature. These coils are connected in series; hence, they are equivalent to a single coil.

Next, we explain the deflection of the armature. When an electric current is given to the coil, the armature becomes an electromagnet. Let assume the armature right end becomes S-pole corresponding to the electric current direction assigned in Fig.1. Then the armature right end is attracted from the upper pole and repelled from the lower pole. Conversely, the armature left end is attracted from the lower pole and repelled from the upper pole. Consequently, the armature receives a counter clock-wise moment (torque) $T_{A}$ as shown in Fig.1. The deflection angle of the armature is determined by this torque, the elastic restoring moment of flexure tube, and fluid dynamic forces due to nozzle flows. In the following, we will discuss the armature torque $T_{A}$.

The magnetic lines generated by the permanent magnets and the coil pass through the gaps between the poles and the armature. Consequently, flux density distributions are formed in and around each gap. The armature torque is determined by the flux density distributions.

The magnetic lines passing through the gap 1 are illustrated in Fig.2. Every magnetic line serves tension and repels each other[2]. The tension on a small elementary surface normal to a magnetic line is the product of Maxwell stress and the area of the elementary surface. Some magnetic lines are pressed out from the gap as shown in Fig. 2. This phenomenon is called fringing[3].

The pressed out magnetic lines reach to the side surfaces of the armature. These pressed out magnetic lines also contribute to the force between the pole and the armature. Therefore, the force between the pole and armature is greater than the surface integral of normal component of the stress on the surface $S_{1 \mathrm{p}}$ that bounds the gap as shown in Fig.2. The total force will be obtained integrating the normal component of Maxwell stress on the plane $S_{1}$ in Fig.2. However, it is difficult to perform this integration. Therefore, we will try to express the torque on the arma-

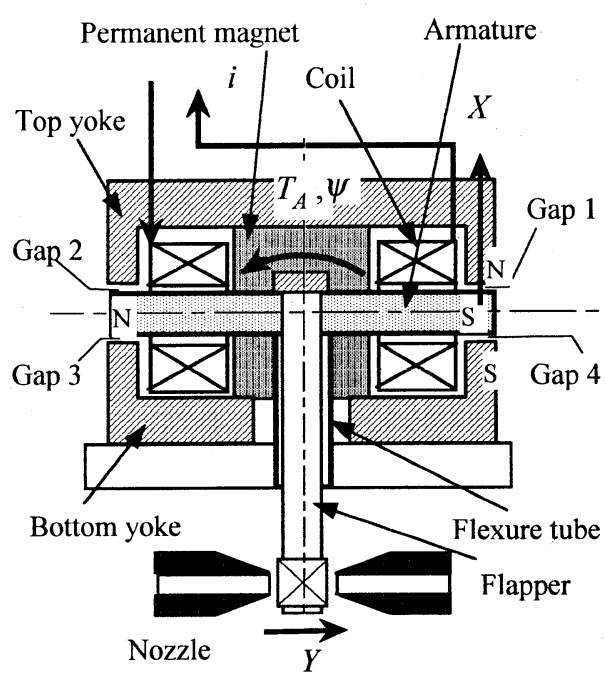

Fig. 1 Torque motor structure

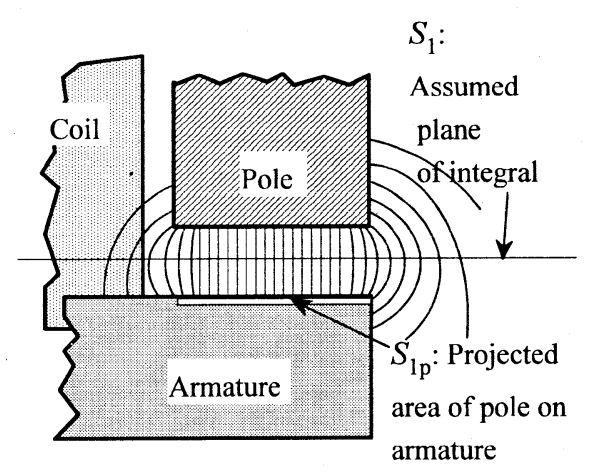

Fig 2. Fringing and magnetic lines

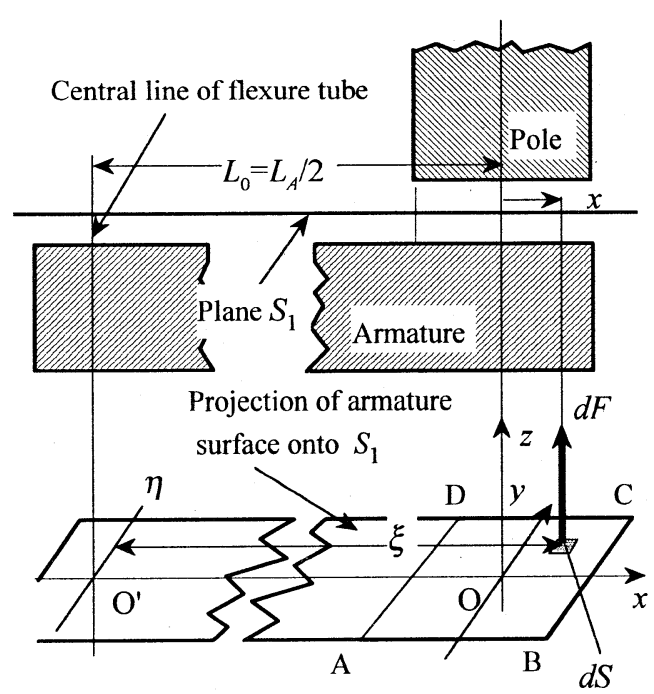

Fig.3 Coordinate system 
ture using the flux densities measured at the centers of each gap.

\section{EXPRESSION OF TORQUE}

For calculation of the torque on the armature, we define coordinates on the plane $S_{1}$ that lies in the midway of surfaces of armature and the pole as shown in Fig. 3. The upper portion of Fig. 3 shows relative position of the pole and the armature; the lower portion of Fig. 3 shows coordinate on the plane $S_{1}$. We can assume that the magnetic lines are normal to $S_{1}$. Denoting the flux density at a point $(x, y)$ as $B(x, y)$, the force acting on the elementary surface $d S$ is expressed by

$$
d F=\frac{B^{2}(x, y)}{2 \mu_{a}} d S .
$$

The moment due to the infinitesimal force with respect to the central line of the flexure tube is

$$
\begin{gathered}
d M=\xi d F=\left(L_{0}+x\right) d F \\
=\frac{\left(L_{0}+x\right) B^{2}(x, y)}{2 \mu_{a}} d S .
\end{gathered}
$$

Integral over the plane $S_{1}$ gives moment due to the gap-1. There are four gaps in the torque motor. Therefore, the torque on the armature is the sum of the contributions of the four gaps:

$$
T_{A}=\iint_{S_{1}} d M+\iint_{S_{2}} d M+\iint_{S_{3}} d M+\iint_{S_{4}} d M .
$$

While the procedure for the evaluation of the moment is the same to all the gaps, we continue our discussion with respect to the gap 1 .

Now, the rectangle $\mathrm{ABCD}$ in Fig. 3 is the projection of the pole surface onto the plane $S_{1}$. The origin $\mathrm{O}$ is the projection of the center of the pole. $B(x, y)$ is considered symmetric with respect to $x$-axis because the geometric structure is symmetric with respect to $x-z$ plane. To obtain a simplified expression for the torque, we assume the flux density is also symmetric with respect to $y$-axis. While the geometric condition is not symmetric with respect to $y$-axis, this assumption should be verified by experimental observation on the flux density distribution. Under this assumption, the moment due to the flux density distribution is simplified as follows:

$$
\begin{aligned}
& M_{1}=\int_{S_{1}} d M=\int_{S_{1}}\left(L_{0}+x\right) d F \\
& =L_{0} \int_{S_{1}} \frac{B^{2}(x, y)}{2 \mu_{a}} d S+\int_{S_{1}} x \frac{B^{2}(x, y)}{2 \mu_{a}} d S
\end{aligned}
$$

$$
=L_{0} \int_{S_{1}} \frac{B^{2}(x, y)}{2 \mu_{a}} d S=L_{0} F_{1}
$$

Here $F_{1}$ is the attractive force between the pole- 1 and the armature[4]. Similar expressions are obtained for the other poles. In addition, the four poles are made to identical shape and configuration. Hence, it holds

$$
A_{1}=A_{2}=A_{3}=A_{4}=A_{g} .
$$

The mean values of the flux density on the planes $S_{i}(i=1$, $2,3,4)$ are

$$
\begin{aligned}
B_{m i}=\frac{1}{A_{i}} \int_{S_{i}} B(x, y) d S= & \frac{\Phi_{i}}{A_{g}} \\
& (i=1,2,3,4) .
\end{aligned}
$$

The integral appeared in (4) yields

$$
\begin{aligned}
& B_{T i}^{2}=\frac{1}{A_{g}} \int_{S_{i}} B^{2}(x, y) d S, \\
&(i=1,2,3,4) .
\end{aligned}
$$

$B_{m i}$ coincides with $B_{T i}$ if $B(x, y)$ takes a constant value over the domain of integration. However, these values are different and difficult to obtain in general. Traditional assumption was to assume the flux as the product of gap area and flux density derived from Ampere's circuit theorem[5]. This assumption is not suitable because the flux density is not uniform on that cross section: the assumption was a cause of discrepancy between theory and design practice.

Let us try to relate these values to an easily observable value. The flux density becomes uniform near the center of each pole of the yokes. Therefore, the flux density at the center is an easily observable value. For calculation of the torque, we define the following dimensionless coefficients:

$$
\left.\begin{array}{l}
B_{m i}=k_{m} B_{C i} \\
B_{T i}=k_{T} B_{C i}
\end{array}\right\}, \quad(i=1, \cdots, 4),
$$

where $B_{C 1}$ is the flux density observed at $\mathrm{O}$ in Fig.3; the other $B_{C i}$ 's are found at equivalent points in the other gaps.

If we know $k_{T}$, we can estimate the torque $T_{A}$ using measured values of $B_{C i}$. Similarly, if we know $k_{m}$, we can correlate the observed value of $B_{C i}$ to the flux $\Phi_{i}$ found by the magnetic circuit analysis.

Since the four gaps are geometrically identical, the value of $k_{T}$ and $k_{m}$ must be identical for each gap. Hence, the torque expressed by ( 3 ) is reduced to 


$$
T_{A}=k_{T}^{2} \frac{A_{g} L_{0}}{2 \mu_{a}}\left(B_{C 1}^{2}-B_{C 4}^{2}+B_{C 3}^{2}-B_{C 2}^{2}\right)
$$

or

$$
T_{A}=\left(\frac{k_{T}}{k_{m}}\right)^{2} \frac{L_{0}}{2 A_{g} \mu_{a}}\left(\Phi_{1}^{2}-\Phi_{4}^{2}+\Phi_{3}^{2}-\Phi_{2}^{2}\right) \text {. }
$$

Thus, the armature torque can be found using the flux obtained by the magnetic circuit analysis.

\section{EXPERIMENT AND DISCUSSION}

\section{Measurement of flux distribution}

Figure 4 shows the experimental rig. The torque motor is fixed on the nozzle block. An input signal voltage is supplied from the signal generator to the servo-amplifier that generates an electric current proportional to the input signal. The electric current excites the coil: the current is fed back to the amplifier so that the current takes no significant delay from the signal despite the inductance of the coil. The phase lag between input signal and the output current is less than five degrees by sinusoidal signal of $10 \mathrm{kHz}$.

Displacement of the flapper is measured at the nozzle center position. An eddy current sensor detects the position of the flapper surface without contact. The displacement sensor is mounted on the $x-y-z$-stage-A in Fig. 4 with that the calibration of the sensor is carried out on the rig.

The flux density distribution is measured using a Hall sensor mounted on another $x-y$-z-stage, the stage-B in Fig. 4. The Hall sensor is made of $G_{A} A_{S}$, a rectangle of $75 \times$ $75 \mu \mathrm{m}$ with $70 \mu \mathrm{m}$ thickness. The sensing tip is embedded under $0.1 \mathrm{~mm}$ from the surface of a plastic slab with thickness $0.25 \mathrm{~mm}$.

Inserting and moving the sensor with aid of the stage-B, we set the measuring plane. Then, manipulating the stage, a point $(x, y)$ is set and the value of flux density at the point is measured. Changing the position of the sensor, the flux density distribution measurement is carried out. The signal of the sensor is amplified with a Gauss meter. Measured values of the coil current, the flapper position and the flux density distribution is stored in a personal computer.

With the configuration of Fig.4, the flux density around gaps 1 and 4 are measured. To measure the flux density around gaps 2 and 3, the torque-motor is turned around together with the nozzle block.

The gap thickness is the most important parameter influencing the flux density. However, measurement of it with a microscope, even when combined with photographs, was not reliable for ten-micrometer level accuracy. After some trials, the following measuring procedure was adopted. First, displacement sensor output voltage at free state of the flapper was recorded. Then, a suitable thickness gage was inserted and the corresponding sensor output was recorded. In the same way, two other

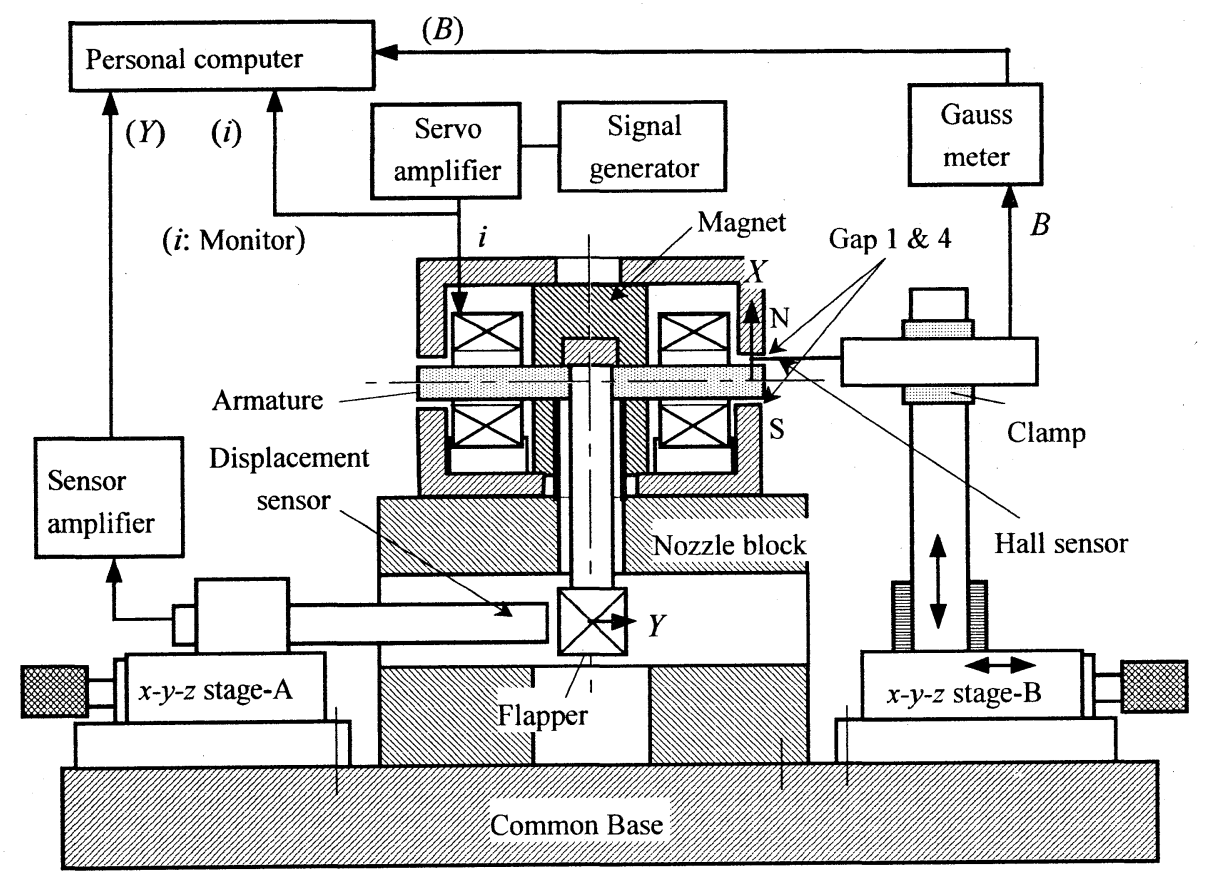

Fig.4 Experimental setup to measure $i, B$ and $Y$ 
thickness gage values were selected and the same procedures were carried out. Thus, we had three sets of values of thick-ness and corresponding sensor output voltages. A plot of the voltage vs. thickness resulted in a straight line. The thickness value corresponding to the sensor output voltage at the free state was read out from the plot. The values thus obtained have shown good repeatability up to the order of one micrometer.

\section{Measured flux density and data processing}

Figure 5 and 6 show the flux distribution on $y$-axis and $x$ axis, respectively. The measurement was made for a few kinds of magnets and under different values of coil current. Through these measurements, it was observed that the shapes of the flux density distributions keep remarkable similarity. Namely, the ratio, "flux density with current/flux density without current," takes almost constant value over the measured area. Another measurement without the permanent magnet, in that condition the magnetic field is generated by coil current, resulted in a similar result. Namely, the ratio, "flux density without permanent magnet/flux density with permanent magnet," takes almost constant value over the measured area. Thus, the measurements suggested that the shape of flux density distribution suffers a minor change from changes of magneto-motive forces.

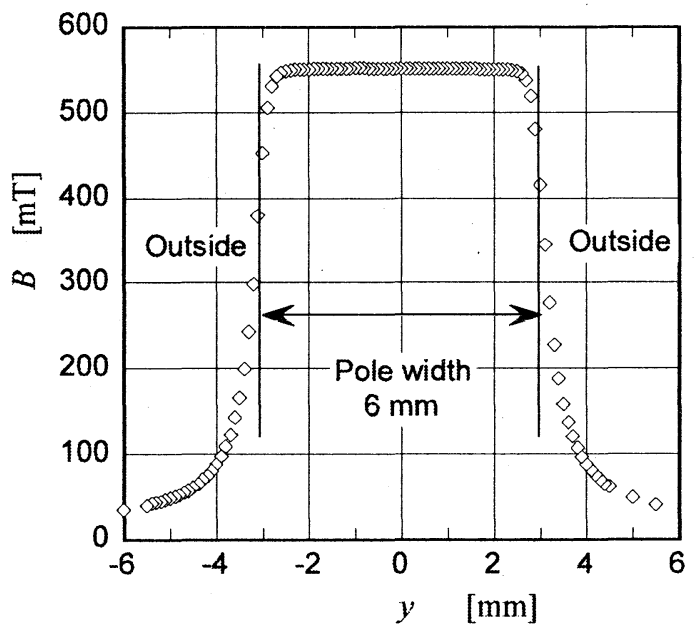

Fig.5 Flux distribution in $y$-direction Magnet: FJ20, Gap4 $=0.512 \mathrm{~mm}, x=0$

Figure 5 shows that the distribution is symmetric with respect to $x$-axis; fringing results in a symmetric change of flux density at both sides of periphery. Figure 6 shows that the distribution is not symmetric with respect to $y$ axis, as the flux density changes gradually at the inside of the torque-motor structure. This gradual change of flux density at the inside is induced by the surface of armature that exists only in the inside of the structure. It will be noteworthy the shape of slope outside of the gap in Fig.6 is similar with the slope shape in Fig.5.

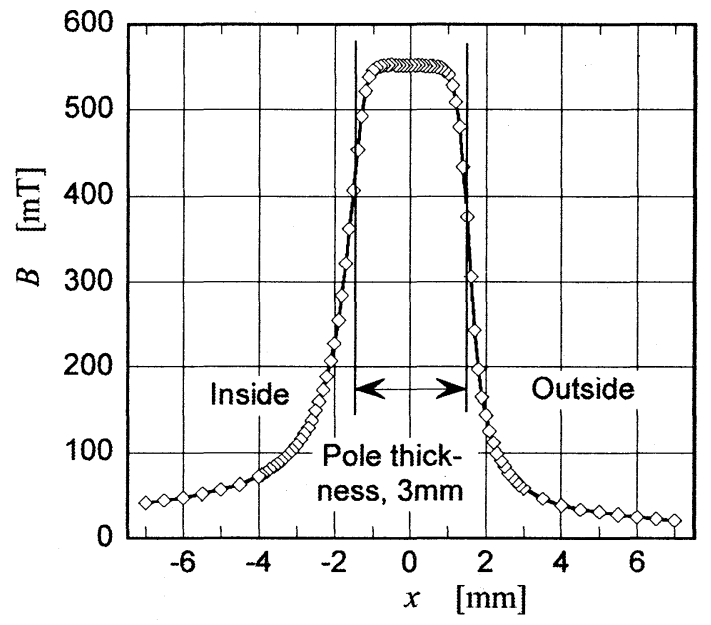

Fig.6 Flux distribution in $\mathrm{x}$-direction Magnet: FJ20, Gap $=0.512 \mathrm{~mm}, \mathrm{y}=0$

\section{Determination of the factors $k_{T}$ and $k_{m}$}

Integrating (6) and (7) on the plane $S$ with respect to the measured flux density, then applying (8), the factors $k_{T}$ and $k_{m}$ can be determined. Actually, however, the plane is partly occupied by the coil. Therefore, a complete measurement was not possible. To compensate the defect of data on this area, the following approximation was applied. Namely, using measured flux distribution along $x$ - and $y$-axis,

$$
\iint_{S_{i}} B(x, y) d S \approx \frac{1}{B_{C i}} \int_{-a}^{a} B(x, 0) d x \int_{-b}^{b} B(0, y) d y .
$$

The limits of integration, $a$ and $b$, were determined observing the measured results. This approximate estimate was compared with the actually measured values in the region $x>0$.

The difference between the measured and estimated values was not negligible magnitude. However, it was still in an acceptable level and we have no effective means that can substitute this method. Hence, we are obliged to adopt this approximation in this article.

Figure 7 shows the two numerical integrals with respect to the measured flux density along $y$-axis:

$$
\begin{aligned}
& I_{B}(y)=\frac{1}{B_{C}} \int_{-y}^{y} B(0, t) d t, \\
& I_{T}(y)=\frac{1}{B_{C}{ }^{2}} \int_{-y}^{y} B^{2}(0, t) d t .
\end{aligned}
$$




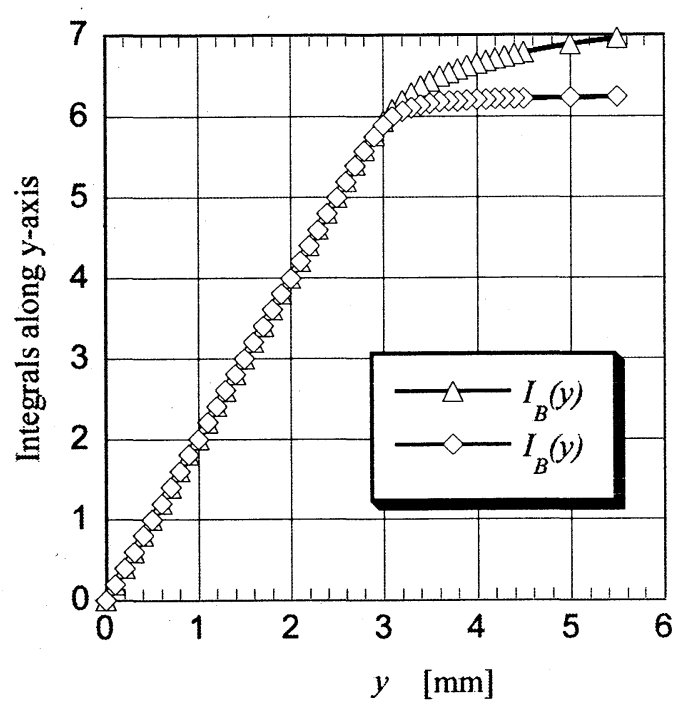

Fig. 7 Integrals along y-axis

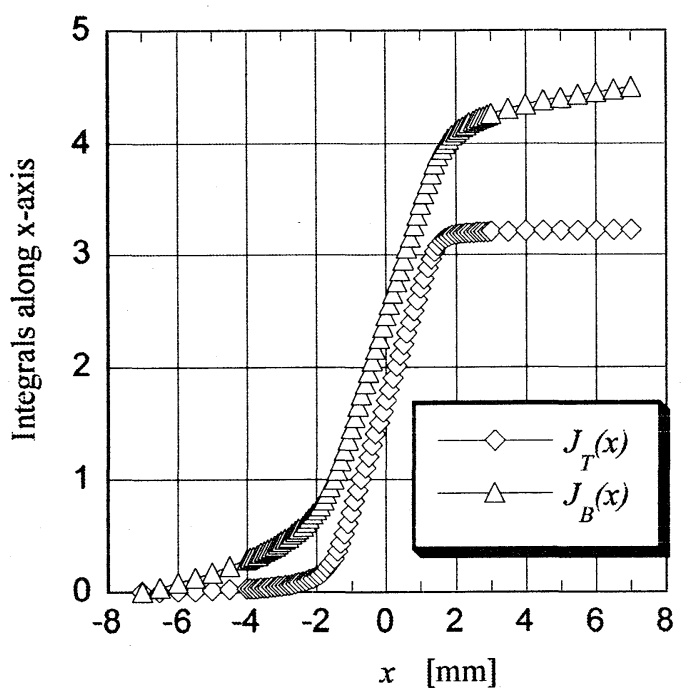

Fig. 8 Integrals along $\mathrm{x}$-axis

Against the pole width $6.00 \mathrm{~mm}$, the final values of $I_{B}(b)$ and $I_{T}(b)$ are about $7.0 \mathrm{~mm}$ and $6.24 \mathrm{~mm}$, respectively. Similarly, Fig. 8 shows the two numerical integrals with respect to the measured flux density along $x$-axis:

$$
\begin{aligned}
& J_{B}(x)=\frac{1}{B_{C}} \int_{-a}^{x} B(t, 0) d t, \\
& J_{T}(x)=\frac{1}{B_{C}{ }^{2}} \int_{-a}^{x} B^{2}(t, 0) d t .
\end{aligned}
$$

A proposal by this article is to estimate the factors with the following equations:

$$
\begin{aligned}
& k_{m}=\frac{J_{B}(a) I_{B}(b)}{4 a b}, \\
& k_{T}{ }^{2}=\frac{J_{T}(a) I_{T}(b)}{4 a b} .
\end{aligned}
$$

The value of $J_{T}(x)$ saturates at about $0.5 \mathrm{~mm}$ away from the side surfaces of pole while the value of $J_{B}(x)$ does not saturate at the point $5 \mathrm{~mm}$ away from the side surfaces of the poles.

Although there is no clear boundary between fringing and leakage, we must limit the range of integral for convenience of torque estimation. The range of integration can be taken, for example, to the point where the integral $J_{T}(x)$ becomes $99 \%$ of the final value. This range can be treated the range of fringing. The flux outside of this range can be considered leakage flux.

As seen from Figs. 7 and $8, J_{B}(x)$ and $I_{B}(y)$ change gradually: hence, it is not convenient to use them to define the range of fringing. Although the curves of $I_{B}(y)$ in Fig. 7 and $J_{B}(x)$ in Fig. 8 do not saturate at this boundary, they show clear change of gradient at the point. This change of gradient supports that the point could be taken as the boundary of the fringing and the leakage.

\section{CONCLUSIONS}

A bridging between the fluxes in the magnetic circuit in the torque-motor and the generating torque is established introducing two coefficients. The first coefficient $k_{m}$ connects the flux and the pole center flux density. The second coefficient $k_{T}$ connects the torque and the pole center flux density. These two factors were estimated based on the measured flux density distribution. Although these coefficients depend on the shapes of the poles, gaps and armature, their values can be used other torque-motors with geometrically similar shapes. Accumulation of this kind of experimental data will improve evaluation of the torque-motor characteristic.

\section{REFERENCES}

1. Urata, E. et al, Development of a Water Hydraulic Servovalve, JSME Internat.J., Ser.B, 1998, 44-2, pp.286-294. 2. Maxwell, J. C., A Treatise on Electricity and Magnetism, (Oxford Classic Series), Clarendon, Oxford, 1998, Vol.2, p. 280

3. Bordea, 1. \& Nazar, S.A., Linear Electric Actuators and Generators, Cambridge Univ. Press, 1997, p.15.

4. Merritt, H.E., Hydraulic Control Systems, John Wiley \& Sons, Inc., 1967, p.184.

5. Urata, E., Study of magnetic circuits for servovalve torque motors, Bath Workshop on PTMC 2000(Edited by Burrows, C.R. and Edge, K.A.), Professional Eng. Pub., London, 2000, pp.269-291. 